\title{
Decision support systems based on scientific evidence: bibliometric networks of invasive Lantana camara
}

\author{
Preet Mishra $^{1}\left(\mathbb{D} \cdot\right.$ Abhishek Prasad $^{2} \cdot$ Suresh Babu $^{3}\left(\mathbb{D} \cdot\right.$ Gitanjali Yadav $^{2}(\mathbb{C}$
}

Received: 3 May 2020 / Accepted: 2 September 2020 / Published online: 10 June 2021

(c) Indian National Science Academy 2021

\begin{abstract}
Extraction and analysis of useful knowledge from the vast amount of relevant published literature can add valuable insights to any research theme or area of interest. We introduce a simplified bibliometric data analysis protocol for gaining substantial insights into research thematics, which can also serve as a handy practical skill for researchers while working from home. In this paper, we provide ways of developing a holistic research strategy using bibliometric-data driven approaches that integrate network analysis and information management, without the need for full paper access. This protocol is a comprehensive multi-modular pathway for analysis of metadata obtained from major scientific publishing houses by the use of a Decision Support System (DSS). A simple case study on the invasive species Lantana camara has been presented as a proof-of-concept to show how one can implement this DSS based protocol. Some perspectives are also provided on how the outcomes can be used directly or scaled up for long term research interventions. We hope that this work will simplify exploratory literature review, and enable rational design of research objectives for scholars, as well as the development of comprehensive grant proposals that address gaps in research.
\end{abstract}

Keywords Decision Support System (DSS) · Text Data Mining (TDM) · Community detection · Bibliometric analysis · Complex networks $\cdot$ Invasive species $\cdot$ Lantana camara

\section{Introduction}

\section{The problem of optimizing knowledge extraction from published literature}

This article is part of the Special Issue: IndianNational Young Academy of Science (INYAS).

Gitanjali Yadav

gy@ nipgr.ac.in

Preet Mishra

preetmishra8@gmail.com

Abhishek Prasad

abhishek1992@nipgr.ac.in

Suresh Babu

suresh@aud.ac.in

1 School of Computational and Integrative Sciences, Jawaharlal Nehru University, New Delhi, India

2 National Institute of Plant Genome Research, New Delhi, India

3 School of Human Ecology, Ambedkar University, New Delhi, India
Informed policymaking necessitates scientific evidence, regardless of system complexity, be it socio-political state priorities during the ongoing novel coronavirus pandemic, funding agency mandates, research strategy development of graduate students, or any other real-world problem. While identifying possible solutions, policymakers require problem-oriented scientific evidence, which in turn, can be understood as a dynamical system possessing a multimodular nature. Accordingly, policy issues and solutions are both embedded in complex, globally interconnected environments that require a thorough analysis of knowledge extracted from available published or bibliometric data. Any evidence derived from incomplete data can be inaccurate, and this brings in two critical aspects associated with usage of vast information:

Firstly, published information can be categorized under the paradigm of big data, possessing all the typical 
characteristics of high variety and volume. Usage of such a large body of information is a complex process. Thus, the analysis of information, if not implemented without due recognition of constraints involved, such as patent information, copyright, open access, metadata, etc. can cause bottlenecks in data adequacy.

Secondly, data insufficiency may arise from the choice of search parameters. Computational tools like citation managers and AI-based search engines harness bibliometric bigdata using author-designated and user-specified "keywords". This step, in turn, controls all the later stages of the analysis; organizing, filtering, classifying, predicting, planning, and so on. If a priori, the keyword is chosen in an ad-hoc or static manner, it may decrease the efficiency of the search process which in turn will strongly impact the outcome. Since both these aspects of the problem are inherent and user-dependent, we suggest a two-pronged strategy of building redundancies and optimizations using network visualizations tools and decision support systems, as described below.

\section{Visualizing bibliography metadata as Networks}

A network approach to decipher large data sets has long been known as one of the best analytical strategies, and this applies equally well for bibliographic information (Shiffrin and Börner 2004), with the added benefits of being able to explore, model and restructure literature metadata to draw insights from both static and dynamic representations of individuals, organizations or themes of research (Newman 2004). High dimensional literature metadata, when visualized efficiently through networks can reveal communities sharing common node or edge attributes in both coarsegrained and fine-grained routines (Babu et al. 2016).

The availability of metadata can often overcome constraints of limited access to full-text and enable one to focus on a lower ease-of-access threshold, i.e. critical information within the title, abstract, and citation. In the paradigm of embedding theory, semantic approaches with use of natural language processing (NLP) efforts and context-based analysis of word embeddings are often very helpful; words embedded within sentences of publications indicate the themes of research and thus the visual analysis of these embeddings may provide proof of concept (Spangler et al. 2014). Such textual embedding of data has high dimensions (Griffiths and Steyvers 2004), and keeping track of the dimensions can be done efficiently through network visualizations of co-occurrences (Tshitoyan et al. 2019). An added benefit is, it can provide us a visual static picture of the flow of ideas and the themes in current research scenarios (Srinivasan 2004). Metadata can thus consist of keywords, title, and abstract, authors' information, publisher and journal information which can all be projected as node attributes, while edges may represent relationships between nodes, such as co-authorship, professional affiliations, or collaborative interactions (Landauer et al. 2004). Co-authorship bibliography networks are undirected, and two of the most informative topological parameters are (a) betweenness centrality, an indicator of hubs in the flow of information, and (b) degree distribution, a measure of the collaborations (Newman et al. 2004).

\section{Decision support system (DSS).}

A vast number of online and downloadable tools are available to perform bibliometric analyses, but as mentioned above, all such software strictly adhere to the concept of Garbage In Garbage out (GIGO), being implementations based on the processing of metadata obtained through userderived "keywords" (Krallinger et al. 2017). If we envisage it as a single process to obtain metadata from a huge database of published articles, it may be possible to optimize critical search parameters, through a Decision Support System (DSS) (Sprague 1980).

Here we present case studies from analysis of invasive plant species bibliographic metadata and share how emerging co-authorship networks can improve and inform decisions, and how diverse network visualizations can be integrated as modules in a DSS. The solution provided from these DSSs must be regarded as partial, being iterative and adaptive, subject to existing constraints in time, rather than being steadfast or all-encompassing. Our main objective is to increase the efficiency of decision making at the initial levels of strategy design, which may contribute to accuracy improvement at later levels in the hierarchy.

\section{Methodology}

\section{Design of the DSS}

Figure 1 depicts a schematic diagram of the suggested decision support system (DSS) for the integration of modules consisting of various bibliography tools, to analyze the metadata of published information from various sources like PUBMED, JSTOR and Web Of Science etc. A-priori the user-selected keyword enters the process and is at the core of advanced search tools for obtaining metadata from various sources alike. This metadata can be analyzed in two distinct ways (dashed line arrows marked as 1 and 2 in Fig. 1); route 1 includes labor intensive reading and analysis of the problem-oriented search results and then updating, on a manual inference basis, the search keyword in the next iteration, a complex and multidimensional landscape that may involve new queries arising from the parsing of metadata and analytical constraints related to accessibility, time availability, feasibility, collaborations, and so on. This juncture can be 


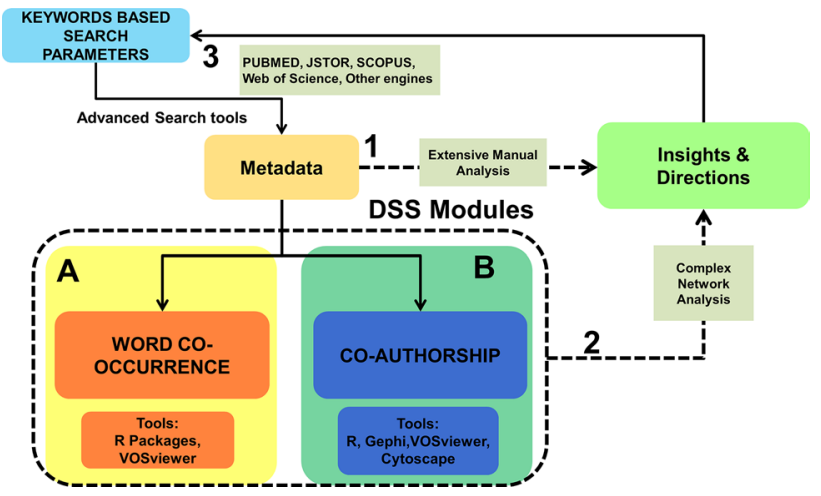

Fig. 1 DSS Schematic. Routes 1 and 2 marks two options to assess bibliometric metadata, with the latter (route marked 2) involving modular analysis of bibliometric networks (modules A, B) as a Decision Support System

identified as the most crucial point in the analysis, where the user has to make decisions regarding the next iteration. Several queries need to be addressed before moving ahead in an exploratory investigation.

For instance, how much information should be sufficient to answer the questions addressed? Do the current citations cover adequate thematic premise? Does the metadata reflect the evolution of subject-specific knowledge through space and time? According to our proposed scheme, route 2 can be used to answer these and other relevant questions through a DSS with two modules A and B, both incorporating diverse aspects of network theory (See Fig. 1).

\section{Bibliography data formats}

Article metadata can provide information at the theme levels thus, it can be related to decisions about choice of keywords and databases to search when doing the literature surveys and concept reviews broadly. Module A in Fig. 1 involves text co-occurrence networks based on the metadata obtained from initial searches. These visualizations can be obtained from the abstract, title, or keywords provided with the published paper. Some of the file formats that are typically used are .txt,.csv,.xml etc. These can then be plugged into the respective tools to obtain relevant visualizations. Some of the tools are mentioned in Fig. 1. Through various algorithms, these tools parse the embeddings of the words in the text and yield mapping of the embeddings, providing users with a clearer picture of the linked concepts, which have been published and latent information that can help users select appropriate keywords for the next iteration.

The metadata obtained from the search can also yield information about the research scenarios at the author level with related information as attributes of the author. The module marked B in Fig. 1 deals with user decisions regarding the choice of the search parameters taking into

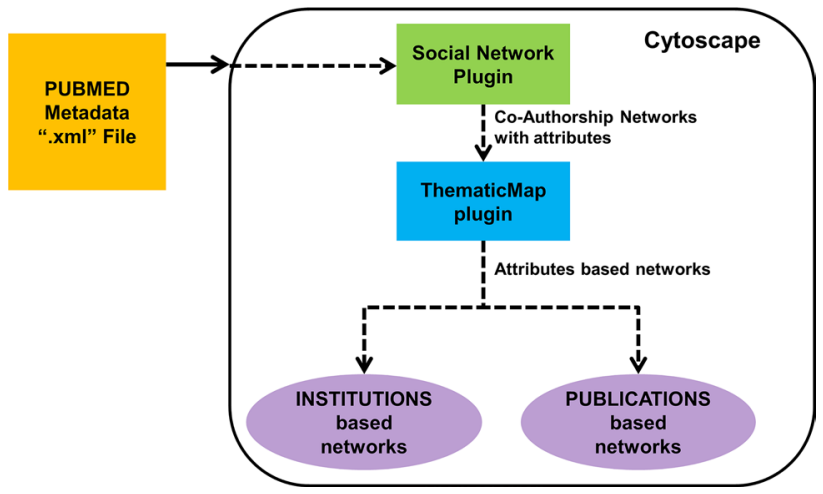

Fig. 2 The flow chart of the methodology adopted for the case study

account author based information from metadata. This module involves tools to create co-authorship networks that can be analyzed regarding not-only decisions about search parameters but also inform the exploratory investigation into research themes by answering questions as to the origins and extent of the problem itself, i.e. who, where, what and when, etc. (Lent et al. 1997). Both DSS modules are highly usercentric and involve open source programming software, with greater levels of accessibility, enabling personalized module design at the user level. Module B depends on the interpretation of the user in a more convoluted way than that involved in module $\mathrm{A}$, and due care must be taken while implementing module $\mathrm{B}$, as well as while deriving interpretations from the DSS outputs at each iteration.

\section{Results}

\section{A case study on Lantana camara}

We present a case study for assisting a graduate student aiming to perform a systematic review of biological invasions, focusing on India's most aggressive alien plant invasive species Lantana camara. Our objective is to roughly get an overview of the status of research in this area and identify major gaps, especially from an Indian context, so that a suitable graduate research strategy can be designed for the next three years, addressing gaps in knowledge.

For this case study, we take route 2 as shown in Fig. 1 and show how combinations of suitable keywords can be developed by multiple iterations of the DSS module, the methodology is summarized as a flowchart in Fig. 2.

For the initial process of obtaining metadata, we focus on the prime objectives of the species in the Indian context. We use PUBMED (https://www.ncbi.nlm.nih.gov/pubmed), the open-source literature database with over 30 million citations for biomedical literature beginning from 1948, life science journals, and online books (https://www.ncbi.nlm.nih. 
gov/books/NBK3827). We used 'advanced search' option and keywords ("lantana"[All Fields]) AND ("camara"[All Fields]) AND ("India"[All Fields]), and this returns over a 100 articles by more than 300 authors. We saved metadata as an XML file, a format compatible with the Cytoscape plugin used in subsequent stages (Shannon et. al., 2003). It may be noted that formats and compatibility between search engines and downstream tools represent a constraint in the visualization process and metadata formats with more flexibility can enable more information to be extracted. For instance, although JSTOR (www.jstor.org) has the largest open collection of published articles (1928 onwards), it does not enable metadata collection, rather each article is downloaded as an individual XML file. We are currently working on developing a tool for collating these files and extracting metadata using $\mathrm{R}$ without losing links between them. It may also be noted that we have chosen a relatively sparse dataset as our example, and we advise due care in the selection of keywords and to correlate these with the number of articles returned. For example, adding the keyword 'invasive' severely reduces the number of articles, whereas removing the connection to India increases the number to beyond 600 .

The XML file feeds into the 'Social Network' plug-in of the most widely used open-source visualization tool Cytoscape (Kofia et al. 2015), yielding the co-authorship bibliography network shown in Fig. 3a. Various attributes of the nodes (authors) can be superimposed onto this network as additional informative layers of color or shape, for example, the institutions they are affiliated to, as well as the theme of research, to obtain visualizations in panels B and $\mathrm{C}$ respectively (Fig. 3). The foremost observed pattern is that Lantana research in India is strongly inclined towards applied phytochemistry, with more than $80 \%$ of authors publishing under this theme (represented by green colored nodes in Fig. 3c). The other notable pattern is that despite very few researchers (only 13\%), being involved in addressing invasion ecology of L.camara in India this is the theme where potential global interest, and possibly funding opportunities,

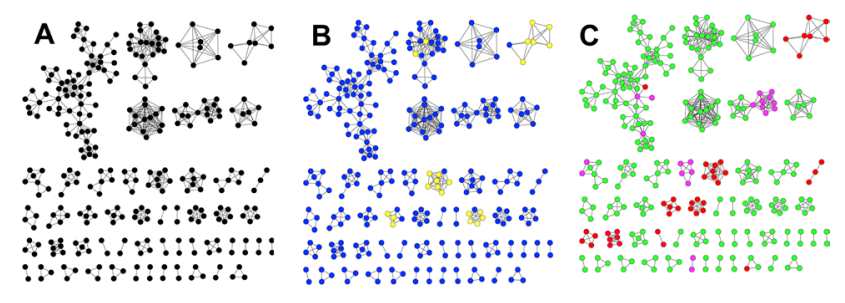

Fig. 3 Co-Authorship Networks overlaid with metadata. a Nodes are authors and edges represent shared publications. b Same as A but color-coded by author affiliations; Blue nodes depict authors from Indian Institutions. c Same as A but color-coded by themes/keywords; 42 Reds (Invasion Ecology), 249 Greens (Applied Phytochemistry), and 15 Pink nodes ( depicting agricultural applications) concerning Lantana camara may be found. This is evident from a comparison of congruent layouts in Figs. 3b and 3c, where the largest proportion of foreign authors (yellow nodes in 3B) are sharing publications with Indian authors under the theme of invasion ecology (represented by red nodes in 3C).

To check if this interpretation is an artifact of network representation, the layouts can be redrawn differently; representing nodes as institutions or publications, rather than individual authors. This has been done in Fig. 4 using the same metadata and color codes as above, with Cytoscape 'ThematicMap' plugin (Shannon et. al., 2003). All attribute networks in Fig. 4 represent edges as authors and the width of these edges depicts the number of authors sharing an affiliation (4A, 4B) or a publication (4C). Colors in Fig. 4a reveal collaborations between Indian (blue) and foreign (yellow) institutions, while color codes in Fig. 4c reveal the theme of the paper. Clearly, the pattern observed in the earlier networks is echoed here as well, with $80 \%$ of the network space representing applied phytochemistry, with the added benefit of being able to map organizations to research themes of interest, such as invasion aspect of Lantana camara.

Additional features of the bibliography networks can be observed in attribute networks, as compared to co-authorship networks; Fig. 4b shows the largest subnetwork of 4A, revealing the most highly associated institutions among existing collaborations, and this subnetwork should be important for decisions regarding setting up new collaborations, or identification of hubs driving the existing body of work, towards bringing in fresh outlook or scope. Nodes in the 4B subnetwork are colored by degree, revealing institutions that have had the maximum collaborative interaction in the area of interest. Interestingly, this subnetwork consists of organizations that are not only related by geographical proximity (like IVRI and CSIR-IHBT both in Palampur, and Doon University, Dehradun) but also connect a wide diversity of expertise (Dept of Biochemistry at Panjab University and Carbohydrate
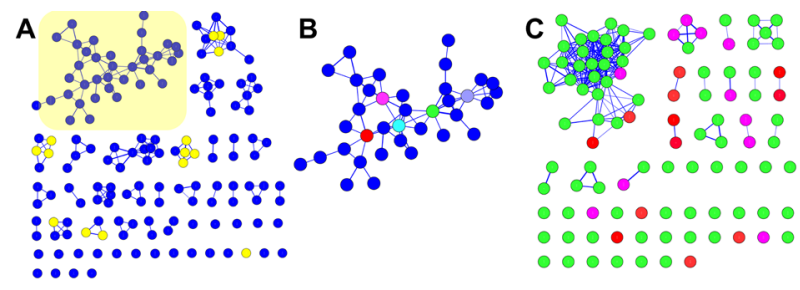

Fig. 4 Attribute networks for L.camara literature (a) Nodes are Indian (blue) or foreign (yellow) institutions while edges represent the number of authors sharing the affiliation. $\mathbf{b}$ The largest subnetwork highlighted in 4A, Top 5 institutes with highest topological centralities are depicted in new colors (see text). c Nodes are papers and the edges represent common authors. Color codes the same as in Fig. 3 
Biotechnology at IIT Guwahati), both being valuable for a new researcher.

Additional features discernable from both sets of networks are related to linkages of research and development units, towards the reduction of multidimensional problems related to research in invasion biology of Lantana camara. For instance, agricultural applications are not very strongly represented in Lantana bibliography networks (only $4 \%$ ), but this is not a niche or gap in research, instead, a reflection of the nature of Lantana camara; it is not an agricultural weed but more widely impacting the conserved/protected areas. As can be seen in Fig. 3c and 4c, the few studies that involve agricultural applications, are also in the area of applied photochemistry, suggesting applicability for Lantana phytochemicals in toxicity assays or weedicide development. The major research gaps from an Indian point of view in this field are that ecology and phytochemistry have not been studied together for this invasive, despite being known to be connected concepts. Another insight from these networks is a large number of disconnected subnetworks, suggesting a relative disconnect between researchers, groups, and institutions working on Lantana camara. This awareness immediately raises the need for a pan-India national network on invasion biology, bridging diverse fields of expertise and knowledge, to address this most aggressive species that has invaded almost all parts of the country.

Strategies to enable more detailed literature review and decision making, would be to expand the search to larger engines like Web of Science and JSTOR, as well as to get a global perspective by dropping the reference to "India" alone. When this search was performed, i.e. dropping the keyword "India" resulted in over 1000 articles in Web of Science while JSTOR cited over 2000 articles in this category. We have combined both searches and are presently developing R scripts to link the metadata from all sources for a more comprehensive and global analysis (MS in preparation). Another option is to perform the next iteration of this DSS by adding more specific theme-specific keywords, phytochemistry being of foremost importance. With the largest number of papers available, new questions could be addressed in phytochemical evolution. Another immediate consequence of this small study would be to expand the search to other invasive alien species, an effort that has already been undertaken in our group. The metadata can also be analyzed using co-occurrence visualization software like VOSviewer (van Eck and Waltman 2010), which forms the other module of the proposed DSS (module B of the DSS as shown in Fig. 1).

\section{Outlook and prospects}

In this work, we have shown that even with a simple DSS on a single iteration, the outcome not only enabled identification and optimization of multiple relevant keywords but also put forth a complete picture of the various aspects of the research theme that emerged outright at the initial stages itself. We also provide ways to scale up and the search operation with additional databases, as well as scaling down with the selection of a range of keywords.

Two courses of action emerge from the DSS and case study presented here. First, a GUI tool could be designed with integrated modules of the above visualization-based analysis as separate programs, which when executed by the user, would act as a source of both quantitative and pictorial depictions of results (Skupin 2004). The process of refining the input to such a tool could then be performed iteratively to obtain precise insights at each step of the procedure. Higher levels of categorization tools exist beyond a high threshold of ease-of-access thus; open-access tools are needed to tackle the issues of a good literature review for usage by a wide spectrum of researchers.

Second, an individual-based support system can be designed subject to the constraint of know-how and ease of access to the above-mentioned network visualizations tools. For example, searching for metadata from webbased databases can be scripted through text and data mining tools like ContentMine, and custom-built in open source platforms like R or Python. Combined with this, the knowledge of advanced network analysis tools like Gephi, Pajek,Neo- $4 \mathrm{j}$ etc. can be used then to analyze the metadata from various perspectives. This will help gather the information that would assist the individual to make a holistic research strategy.

One important aspect often overlooked in bibliography research is the identification of authors who have contributed fairly important research findings, but with fewer collaborations and minimum publications. Measuring author contribution and impact is an interesting theme and has been the subject of many scholarly debates and discussions over the years, mainly because the most commonly used author-metrics and citation indices are biased towards greater numbers of publications, while impact factor indices are increasingly being recognized as representations of journal brand name, rather than of the work. One way of identifying such authors would be to use network centralities that are able to capture features, such as, for example, 'transitivity', or 'betweenness centrality' measures, which could, in principle, indicate nodes (papers) that are "fairly important" i.e. have fewer edges (authors) but that join dense hubs. These could be authors who connected two previously distinct schools of thought, or found common 
ground across multiple existing sub-themes of a larger domain and so on.

In summary, networks do have the patterns, the question is to identify these and we hope that our work will pave the way for new scientific-evidence based insights, policy decisions, and future directions through streamlined network analytics. In exceptional circumstances such as the present Covid-19 lockdown, a lot of review work and meta-analysis is being carried out globally. With a few strategic interventions, many of these in silico efforts could provide insights into gaps and opportunities in research thematic across a range of disciplines.

Acknowledgements AP acknowledges CSIR Junior Research Fellowship. GY acknowledges SERB Grant ID EMR/2016/006486, GCRFBBSRC Grant ID BBSRC BB/P027970/1TIGR2ESS (Transforming India's Green Revolution by Research and Empowerment for Sustainable food Supplies), and NIPGR support. PM acknowledges JNU and UGC for support. SB acknowledges support from AUD and CUES during PM's internship for this work.

Author contributions SB and GY designed the study and proof of concept. PM and AP collected the data. PM built the DSS and performed the analysis. All authors contributed to the preparation of the manuscript.

\section{Declarations}

Conflict of interest The authors declare no conflicts of interest.

\section{References:}

Babu, S., Yadav, G.: Co-Authorship networks among DRDO life science scientists. Defence Life Sci. J. 1, 188-191 (2016)

Griffiths, T.L., Steyvers, M.: Finding scientific topics. Proc. Natl. Acad. Sci. U.S.A. 101(suppl 1), 5228-5235 (2004)

Kofia, V., Isserlin, R., Buchan, A.M., Bader, G.D.: Social Network: a Cytoscape app for visualizing co-authorship networks. F1000Research 4, 481 (2015)
Krallinger, M., Rabal, O., Lourenço, A., Oyarzabal, J., Valencia, A.: Information retrieval and text mining technologies for chemistry. Chem. Rev. 117, 7673-7761 (2017)

Landauer, T.K., Laham, D., Derr, M.: From paragraph to graph: latent semantic analysis for information visualization. Proc. Natl. Acad. Sci. U.S.A. 101(suppl 1), 5214-5219 (2004)

Lent, B., Agrawal, R., Srikant, R.: Discovering trends in text databases. In: Proceedings of KDD, International Conference on Knowledge Discovery, NewPort Beach CA, pp 227-230 (1997)

Newman, M.E.J.: Coauthorship networks and patterns of scientific collaboration. Proc. Natl. Acad. Sci. U.S.A. 101(suppl 1), 5200-5205 (2004)

Newman, M.E.J., Girvan, M.: Finding and evaluating community structure in networks. Phys. Rev. E 69, 026113 (2004)

Shannon, P., Markiel, A., Ozier, O., Baliga, N.S., Wang, J.T., Ramage, D., Amin, N., Schwikowski, B., Ideker, T.: Cytoscape: a software environment for integrated models of biomolecular interaction networks. Genome Res. 13, 2498-504 (2003)

Shiffrin, R.M., Börner, K.: Mapping knowledge domains. Proc. Natl. Acad. Sci. U.S.A. 101(suppl 1), 5183-5185 (2004)

Skupin, A.: The world of geography: visualizing a knowledge domain with cartographic means. Proc. Natl. Acad. Sci. U.S.A. 101(suppl 1), 5274-5278 (2004)

Sprague, R.H.: A framework for the development of decision support systems. MIS Q. 4, 1-26 (1980)

Spangler, S., Wilkins, A.D., Bachman, B.J., Nagarajan, M., Dayaram, T., Haas, P., Regenbogen, S., Pickering, C.R., Comer, A., Myers, J.N., Stanoi, I., Kato, L., Lelescu, A., Labrie, J.J., Parikh, N., Lisewski. A.M., Donehower, L., Chen, Y., Lichtarge, O.: Automated hypothesis generation based on mining scientific literature In: Proc. 20th ACM SIGKDD Intl Conf. Knowledge Discovery and Data Mining 1877-1886 (2014)

Srinivasan, P.: Text mining: Generating hypotheses from MEDLINE. J. Am. Soc. Inf. Sci. 55, 396-413 (2004)

Tshitoyan, V., Dagdelen, J., Weston, L., Dunn, A., Rong, Z., Kononova, O., Persson, K.A., Ceder, G., Jain, A.: Unsupervised word embeddings capture latent knowledge from materials science literature. Nature 571, 95-98 (2019)

van Eck, N.J., Waltman, L.: VOSviewer, a computer program for bibliometric mapping. Scientometrics 84, 523-538 (2010) 